\section{FRI0266 COMPARISON OF CLINICAL AND SEROLOGICAL CHARACTERISTICS BETWEEN PATIENTS WITH SYSTEMIC LUPUS ERYTHEMATOSUS WITH AND WITHOUT ASSOCIATED JACCOUD ARTHROPATHY}

A. Alía Jiménez, W.A. Sifuentes Giraldo, L. Villalobos Sánchez, M.J. García Villanueva, A. Zea Mendoza. Rheumatology, Ramon y Cajal University Hospital, Madrid, Spain

Background: Jaccoud arthropathy (JA) is a chronic non-erosive reversible joint disorder commonly associated with systemic lupus erythematosus (SLE), and occurs in roughly $5 \%$ of all cases. Some studies suggest different profiles of clinical and immunological features between SLE patients with or without JA.

Objectives: To compare the profile of clinical and serological manifestations of patients with SLE with and without JA followed in a tertiary care hospital of Madrid. Methods: We performed a retrospective observational study of a cohort of patients diagnosed with SLE (4 or more ACR criteria) between June 1977 and December 2015. The variables evaluated included demographics, clinical, analytical and radiological manifestations. The definition of JA was based on the presence of clinical criteria (reversible joint deformities) and absence of radiographic erosions typical of rheumatoid arthritis. Statistical analysis was performed using SPSS software version 21.0

Results: We included 108 patients representing a sample of $24 \%$ of the total number of patients with SLE treated at our center during that period. The majority of patients were women (89.8\%), mean age at diagnosis was $30 \pm 12.29$ years (range: $7-75$ ) and duration of disease was 127 months (range: 2-411). Thirteen patients $(12.03 \%)$ had findings compatible with JA. There were no significant differences in age, sex or race, but the duration of disease was higher in JA patients ( 190 vs. 118.2 months, $p=0.0299$ ). There were significant differences in the presence of malar rash $(p=0.0009)$, photosensitivity $(p=0.0050)$, oral ulcers $(p=0.0032)$ and pericarditis $(p=0.000001)$, which were more frequent in patients without JA, but arthritis, nephritis, pleuritis, seizures, psychosis, Raynaud's phenomenon and antiphospholipid syndrome had a similar distribution between both groups. Among the immunological features, no significant difference was found in relation to hemolytic anemia, lymphopenia, thrombocytopenia, ANA, antiENA, anti-DNA, anticardiolipin, anti- $\beta 2$ glycoprotein I and lupus anticoagulant, but leukopenia was also more frequent in patients without JA $(p=0.0041)$.

Conclusions: In the analyzed sample of patients in our center JA was a relatively frequent finding and was associated with a longer duration of the disease. It was not possible to corroborate other JA associations suggested in previous studies such as a lower frequency of lupus nephritis or major secondary antiphospholipid syndrome, probably due to limited sample size, but there are also other studies that do not demonstrate significant differences in relation to clinical and serological findings in patients with SLE with JA with respect to those who do not present it. Studies involving a larger number of patients with JA associated with SLE are required in order to better identify these differences, so a multicenter initiative could be of great help.

Disclosure of Interest: None declared

DOI: 10.1136/annrheumdis-2017-eular.6980

\section{FRI0267 HEMOPHAGOCYTIC SYNDROME IN PATIENTS FROM SLE REGISTRY FROM THE SPANISH SOCIETY O RHEUMATOLOGY (RELESSER)}

A. Lois-Iglesias ${ }^{1}$, I. Rua-Figueroa ${ }^{2}$, F. deToro-Santos ${ }^{1}$, A. Zea-Mendoza ${ }^{3}$, J. López-Longo ${ }^{4}$, J. Calvo ${ }^{5}$, M. Galindo ${ }^{6}$, E. Uriarte-lsacelaya ${ }^{7}$,

J. Pego-Reigosa ${ }^{8,9}$ on behalf of EASSER-RELESSER. ${ }^{1}$ University Hospital $A$

Coruña, A Coruña; ${ }^{2}$ Hospital Universitario Dr Negrin, Gran Canaria; ${ }^{3}$ University

Hospital Ramón y Cajal; ${ }^{4}$ HGUGregorio Marañón, Madrid; ${ }^{5}$ Hospital, Araba;

6 University Hospital 12 de Octubre, Madrid: 7 University Hospital Donostia,

Donostia; ${ }^{8}$ Hospital Vigo; ${ }^{9}$ IISGS, Vigo, Spain

Background: Systemic Lupus Erythematosus (SLE) is an autoimmune systemic rheumatic disease that, in our area, presents hematologic manifestations in approximately $70 \%$ of cases ${ }^{1}$. Some of them are very rare so there are no large series whose analysis could provide relevant information.

Objectives: To study the characteristics of patients with Hemophagocytic Syndrome (HS) in a large sample of SLE patients.

Methods: SLE patients from RELESSER database were studied. We analysed SLE manifestations present at 12 different domains (mucocutaneous, renal, musculoskeletal, constitutional, hematologic, vascular, cardiac, respiratory, neuropsychiatric, gastrointestinal, ophthalmic and serological) before, during and after HS diagnosis and until the last available assessment. We also studied activity (SELENA-SLEDAI) and damage (SLICC/ACR DI) indices at those moments. We evaluated the treatment received, HS recurrences and the number of deaths by this entity.

Results: 3,656 SLE patients ( $\geq 4$ ACR criteria) from 45 Rheumatology Units across Spain were studied. Seven patients $(<0.5 \%)$ with HS were identified. $71.4 \%$ were women, with a mean age ( \pm S.D. $)$ at SH diagnosis of $35.1( \pm 17.1)$ years. In 5 of the 7 cases the HS occurred 115.5 ( \pm 162.9) months after SLE diagnosis. In the other 2 cases the diagnosis of both entities was simultaneous. The main triggers of HS were infections, followed by SLE activity flares. At the time of HS diagnosis, they had high SLE activity with a mean SLEDAI score of $13.1( \pm 11.3)$ and $1.4( \pm 2.3)$ SDI score
Clinically, $100 \%$ of the patients presented fever and alterations of the liver profile, $85.8 \%$ cytopenias and $71.5 \%$ dermatological manifestations. Respiratory manifestations and hemolytic anemia were present in $57.2 \%$ of the cases, both; lymph nodes and coagulopathy in $42.9 \%$. Hepatomegaly was detected in $28.6 \%$, as well as neuropsychiatric, digestive and renal manifestations. Splenomegaly was detected in $14.3 \%$. The mean hemoglobin level was $8.6( \pm 1.1) \mathrm{g} / \mathrm{dl}$, platelets $85,585( \pm 83,390) / \mathrm{mm}^{3}$, ferritin $7,410( \pm 6,470) \mathrm{ng} / \mathrm{ml}$ and triglycerides 404.7 $( \pm 235.6) \mathrm{mg} / \mathrm{dl}$. All patients were admitted and undergonebone marrow study, requiring a mean of $2.2( \pm 1.5)$ treatment lines, using $2.8( \pm 1.7)$ drugs. One patient died during the HS episode by the HS itself and another 2 patients had 2 and 3 recurrences, respectively.

The following table shows the characteristics of each patient.

\begin{tabular}{|c|c|c|c|c|c|c|c|}
\hline & Patient 1 & Patient 2 & Patient 3 & $\begin{array}{l}\text { Patient } 4 \\
\text { Pat }\end{array}$ & Patient 5 & Patient 6 & $\begin{array}{l}\text { Patient } 7 \\
\end{array}$ \\
\hline 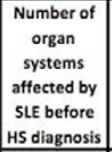 & 9 & 4 & 5 & 5 & 6 & $\begin{array}{c}\text { Simultaneous } \\
\text { diagnosis of } \\
\text { SLE and SH }\end{array}$ & $\begin{array}{l}\text { Simultaneous } \\
\text { diagnosis of } \\
\text { SLE and SH }\end{array}$ \\
\hline 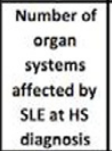 & 4 & 1 & 2 & 4 & 2 & 4 & 3 \\
\hline \begin{tabular}{|c|}
$\begin{array}{c}\text { Number of } \\
\text { organ } \\
\text { systems } \\
\text { affected by } \\
\text { sLE until last } \\
\text { assessment }\end{array}$ \\
ass
\end{tabular} & Died & 2 & 2 & $\begin{array}{c}\text { Follow-up } \\
\text { lost }\end{array}$ & 1 & 5 & 1 \\
\hline \begin{tabular}{|c|} 
SLEDAl//SUC \\
C-ACR DI at \\
HS diagnosis
\end{tabular} & $4 / 6$ & $4 / / 0$ & $5 / 0$ & $29 / / 3$ & $4 / 1$ & $25 / 10$ & $21 / 10$ \\
\hline $\mid \begin{array}{c}\text { SLEDAl//SUC } \\
\text { C.ACR DI 1 } \\
\text { year after HS }\end{array}$ & $*$ & $0 / / 0$ & $0 / / 0$ & * & $2 / 1$ & $0 / 10$ & $2 / 13$ \\
\hline $\begin{array}{c}\text { SLEDAl//SUC } \\
\text { CACR Dl at } \\
\text { last } \\
\text { assessment }\end{array}$ & * & $0 / / 0$ & $0 / / 0$ & * & $0 / 1$ & $2 / 10$ & $2 / 13$ \\
\hline \begin{tabular}{|c|}
$\begin{array}{c}\text { Number of } \\
\text { treatment } \\
\text { lines }\end{array}$ \\
\end{tabular} & 1 & 0 & 3 & 2 & 2 & 4 & 3 \\
\hline \begin{tabular}{|c|} 
Number \\
\\
Treatments \\
administered
\end{tabular} & $\mathrm{GC}$ and $\mathrm{CsA}$ & 0 & \begin{tabular}{|c|}
5 \\
GCs, \\
etoposide, iv \\
Ig, CsA, \\
platelets, red \\
cells \\
\end{tabular} & \begin{tabular}{|c|}
3 \\
GC. CYP and \\
iv Ig
\end{tabular} & \begin{tabular}{|c|}
2 \\
Amphoterici \\
$\mathrm{n} \mathrm{B}$, \\
miltefosine
\end{tabular} & $\begin{array}{c}4 \\
\text { GC, iv Ig, } \\
\text { CsA, MM }\end{array}$ & \begin{tabular}{|c|}
4 \\
GC, CS A, \\
anakinra and \\
CYP
\end{tabular} \\
\hline Relapses & 0 & 2 & 0 & 3 & 0 & 0 & 0 \\
\hline Deaths & Yes & No & No & No & No & No & No \\
\hline \begin{tabular}{|c|}
$\begin{array}{c}\text { Follow-up } \\
\text { time } \\
\text { (months) }\end{array}$ \\
\end{tabular} & Died & 45 & 80 & $\begin{array}{c}\text { Follow-up } \\
\text { lost }\end{array}$ & Unknown & 26 & 24 \\
\hline
\end{tabular}

GC:Glucocorticoids, iv Ig: intravenous immunoglobulins,

CYP: Cyclophsphamide, CSA:Ciclosporine A, MM: Mycophenolate mofetil

Conclusions: HS is a rare life-threatening SLE manifestation. It must be suspected in SLE patients with persistent fever who do not respond to antibiotics, cytopenias and evidence of multiorgan involvement. Relapses and death are common in HS associated to SLE.

References:

[1] Pego-Reigosa JM, Rua-Figueroa I, Lopez-Longo FJ, Galindo-Izquierdo M, Calvo-Alen J, Olive-Marques A, et al. Analysis of disease activity and response to treatment in a large Spanish cohort of $s$ with systemic lupus erythematosus. Lupus 2015;24:720-9.

Acknowledgements: Work supported by Spanish Society of Rheumatology, FIS/ISCIII (PI11/02857), BIOCAPS from the European Union 7th Framework Programme/REGPOT-2012-2013.1 (316265), GSK, Roche, Novartis, UCB.

Disclosure of Interest: None declared

DOI: 10.1136/annrheumdis-2017-eular.2542

\section{FRI0268 ULTRASOUND VS. MAGNETIC RESONANCE IMAGING IN DIAGNOSIS OF PRIMARY SJÖGREN'S SYNDROME}

A. Haidmayer ${ }^{1}$, M. Magyar ${ }^{2}$, M.H. Stradner ${ }^{1}$, T. De Zordo ${ }^{3}$, A. Lutfi ${ }^{4}$, B. Unger ${ }^{2}$ W.B. Graninger ${ }^{1}$, J. Hermann ${ }^{1}$, C. Dejaco ${ }^{1} .{ }^{1}$ Department of Internal Medicine; ${ }^{2}$ Department of Neuroradiology, Univ. Klinik Graz, Graz; ${ }^{3}$ Department of Radiology, Medical University of Innsbruck, Innsbruck; ${ }^{4}$ Department of Radiology, Univ. Klinik Graz, Graz, Austria

Background: Salivary gland (SG) biopsy is the gold standard for the diagnosis of primary Sjögren's syndrome (pSS). Ultrasound (US) and magnetic resonance imaging (MRI) of the SG are emerging techniques. The comparison of both imaging methods for diagnosis and assessment of functional limitation is yet not well investigated. 
Objectives: To assess the accuracy of US and MRI and to define possible cutoff values for the diagnosis of pSS.

Methods: Twenty-three patients with pSS according to AECG criteria and typical histology of the SG biopsy and 12 patients with sicca-syndrome and normal SG histology were included in the study. Two experienced ultrasound experts (C.D. $6 y$, T.D.Z. 5y) did ultrasound of the SG using a B-mode score (0-48 points [1]) and real-time sonoelastography (RTS; $0-16$ pts [2]). Morphology of the parotid glands was also assessed by MRI (0-12 pts). We obtained clinical data (Creactive protein (CRP), antinucleary-antibodies (ANA), Ro-/La-antibodies, Gamma globulins, patient questionaires ESSDAI and SSDI). The statistically analysis was carried out using Kolmogorov-Smirnov-test, student's t-test, or Man-Whitney-Utest. Correlations were performed using Spearman-rang-correlations.

Results: Patients with pSS had significantly higher B-mode- (average $=25$ [2-44] vs. 9 [1-20], $p<0.001)$ and RTS-scores $(6.5$ [2-13] vs. 4 [1-9], $p<0.001)$ than sicca-patients. The same was also found for MRI-assessment $(6,96$ vs. 2,33, $\mathrm{p}=0.001)$. In a Spearman rank correlation, clinical parameters were linked to the imaging techniques The B-mode showed significant inverse correlations with the Saxon-test $(r=-0.505, p=0.002)$ and a positive correlation with MRI $(r=0.792$, $p<0.0001)$. No correlation was found for the activity scores ESSDAI $(p=0.221)$ or SSDI $(p=0.219)$. The MRI score had an inverse correlation with the Saxon-test $(r=-0.523, p=0.001)$. Both imaging techniques showed no relationship with ESR or CRP. We also generated ROC curves of both imaging methods to define possible cutoff values for the diagnosis. For B-Mode we would recommend a value of 12 points (sensitivity $82,6 \%$ and specificity $91,7 \%$ ) and for MRI 3.5 points $(78,3 \%$ and $91,7 \%)$.

Conclusions: Sonography and MRI detected typical morphological changes in the SG of pSS with high sensitivity and specificity. Both methods could become valuable tools for the diagnosis of pSS.

References:

[1] Hocevar, Ambrozic A, Rozman B, Kveder T, Tomsic M. Ultrasonographic changes of major salivary glands in primary Sjogren's syndrome. Diagnostic value of a novel scoring system. Rheumatology (Oxford). 2005;44(6):768-72.

[2] Rubaltelli, Corradin S, Dorigo A, Stabilito M, Tregnaghi A, Borsato S, Stramare R. Differential diagnosis of benign and malignant thyroid nodules at elastosonography. Ultraschall Med. 2009;30(2):175-9.

Disclosure of Interest: None declared

DOI: 10.1136/annrheumdis-2017-eular.6570

\section{FRI0269 "IF IT'S NOT MULTIPLE SCLEROSIS, LOOK FOR A CONNECTIVE TISSUE DISEASE": ATYPICAL DEMYELINATING DISORDERS REFERRED TO A TERTIARY RHEUMATOLOGY CENTRE}

A. Fanouriakis ${ }^{1}$, K. Voumvourakis ${ }^{2}$, M. Papathanasiou ${ }^{3}$, T. Doskas ${ }^{4}$, T. Karageorgas ${ }^{1}$, D. Tseronis ${ }^{1}$, D. Kassara ${ }^{1}$, A. Erden ${ }^{5}$, P. Katsimbri ${ }^{1}$, D. Boumpas ${ }^{1} .{ }^{1}$ Rheumatology and Clinical Immunology, 4th Department of Internal Medicine; ${ }^{2}$ 2nd Department of Neurology; ${ }^{3}$ 2nd Department of Radiology, "Attikon" University Hospital; ${ }^{4}$ Department of Neurology, Athens Naval Hospital, Athens, Greece; ${ }^{5}$ Department of Rheumatology, Hacettepe University, Faculty of Medicine, Ankara, Turkey

Background: Central nervous system (CNS) demyelination is the hallmark of multiple sclerosis (MS), but may rarely occur in connective tissue diseases (CTD), mainly systemic lupus erythematosus (SLE) and antiphospholipid syndrome (APS).

Objectives: To examine whether patients with demyelinating syndrome and atypical features for MS may exhibit an underlying connective tissue disease. Methods: Patients referred to the Rheumatology and Clinical Immunology Unit of "Attikon" University Hospital for a demyelinating disorder characterized as "atypical for MS" following neurologic evaluation, were included in the study. All cases were discussed with a neurologist with expertise in MS and CNS magnetic resonance imaging were assessed by an experienced neuroimagist. Cases included either i) a clinical syndrome suggestive of a demyelinating process (demyelinating optic neuropathy, myelopathy, internuclear opthalmoplegia etc.) and/or ii) abnormal imaging of the central nervous system (CNS) with features suggestive of demyelination (ie. presence of supra- and infratentorial lesions, periventricular location, gadolinium enhancement, Dawson's fingers, hypointense T1-lesions ("black holes"), "dirty" white matter). Patients with brain lesions in MRI more compatible with non-specific white matter hyperintensities (WMHI, ie. of possible ischemic/microvascular etiology) were excluded from the study.
Results: 21 patients were included in the study [all women, mean (SD) age at first neurologic manifestation 37.7 (10.6) years]. 17 patients had MRI findings of a demyelinating process in the CNS (brain lesions only in 7 , spinal cord lesions only in 4 and both brain and spinal cord lesions in 5 patients); WMHI or normal findings were found in 4 patients with optic neuritis. In this selected group of patients, detailed rheumatologic evaluation revealed clinical and laboratory findings suggestive of a CTD in all patients. The most common findings were: arthritis (80.5\%), Raynaud's phenomenon (42.8\%), photosensitivity (38.1\%), malar rash/erythema (33.3\%), livedo reticularis $(23.8 \%)$ and leukopenia $(23.8 \%)$. Antinuclear antibodies were positive in two-thirds of patients $(66.7 \%)$, anti-Ro/La in $19.0 \%$ and aPL only in one patient (4.8\%). Final diagnoses were undifferentiated CTD, in 10 patients, frank SLE in 9 patients, primary obstetric APS and RA, in one patient each. After a median (range) follow-up of $3(1-14)$ years, three patients fulfilled the criteria for MS and received MS-specific therapy, thus were subsequently classified as having an overlap of two diseases.

Conclusions: CNS lesions suggestive of demyelination on MRI must be distinguished from non-specific lesions of microvascular etiology. In cases of demyelinating syndromes not fulfilling criteria for MS, features of an underlying CTD, suggestive of SLE, are frequently found. A small percentage of patients may go on to develop frank MS during follow-up, thus longitudinal monitoring is necessary.

References:

[1] Differential diagnosis of suspected multiple sclerosis: a consensus approach. Miller DH, Weinshenker BG, Filippi M, et al. Multiple Sclerosis 2008; 14: 1157-1174.

Disclosure of Interest: None declared

DOI: 10.1136/annrheumdis-2017-eular.6438

\section{FRI0270 URINARY AND SERUM NEUTROPHIL GELATINASE- ASSOCIATED LIPOCALIN AS A BIOMARKER IN INDIAN CHILDREN WITH SYSTEMIC LUPUS ERYTHEMATOSUS: RELATION TO RENAL INVOLVEMENT AND OVERALL DISEASE ACTIVITY}

B. Abujam ${ }^{1}$, S. Singh ${ }^{2} .{ }^{1}$ Pediatric Allergy Immunology unit, Post Graduate Institute Medical Education Research: ${ }^{2}$ PGIMER, Chandigarh, India

Background: Renal involvement often results in long-term morbidity in childhood Systemic Lupus Erythematosus (cSLE). Neutrophil gelatinase associated lipocalin (NGAL) has been shown to be a reliable structural biomarker for the early diagnosis of kidney injury in many clinical scenarios.

Objectives: This study aimed to detect levels of urinary and serum NGAL and changes in flare and improvement on longitudinal follow up in CSLE in a real world clinical scenario.

Methods: Children $<14$ years of age attending the Pediatric Rheumatology clinic, fulfilling the 1997 SLE criteria were recruited. Urine and serum samples were collected during routine clinical review and SLE Disease Activity Index (SLEDAI) assessed. Children were divided into 3 categories, active renal, active non-renal and inactive lupus. Active lupus was defined as SLEDAI $>4$. Urinary and serum levels of NGAL (UNGAL; sNGAL) were assessed by ELISA. Urinary values were normalized for urinary spot creatinine. In addition, some patients were longitudinally followed up and resampled when their disease activity changed.

Results: The study included 122 ( $\mathrm{F}: \mathrm{M}=91: 31$ ) children, 54 had active renal,14 had active non-renal and 54 had inactive disease. Median (IQR) age was 8.8 (6.5-10.7) years and disease duration was 10 (3-24) months.26 children with nephrotic syndrome and 49 age and gender matched healthy controls were also recruited. Children with active renal lupus had significantly higher UNGAL as compared to other categories.Although SNGAL was significantly higher in active renal as compared to inactive lupus, there was no difference between renal and non-renal active lupus (Table 1). On longitudinal follow up, UNGAL levels increased markedly prior to a flare, significantly higher in renal compared to a non-renal flare $(p<0.05)$. On the other hand, active lupus children had a significant fall in their uNGAL on follow up. Their was good correlation between change in SLEDAI and change in absolute uNGAL levels $(r=0.84, p<0.01)$. Overall, on ROC analysis, UNGAL classified active renal versus active non-renal and inactive combined with an AUC of $0.986(95 \% \mathrm{Cl} 0.972-1.0)$. The sensitivity and specificity of a UNGAL cutoff off value of $25750 \mathrm{ng} / \mathrm{ml}$ was 96.3 and $91.2 \%$ respectively.

Conclusions: Urinary NGAL is a sensitive marker of renal involvement in SLE disease activity and can also be a reliable tool for monitoring renal disease activity changes.

Abstract FRI0270 - Table 1

Inactive cSLE $(\mathrm{n}=54)$

Active renal cSLE $(n=54)$

Active non-renal cSLE $(n=14) \quad$ Healthy controls $(n=49) \quad$ Nephrotic controls $(n=26)$

Demographics

Age (years) (mean $\pm S D)$

Age at onset (years) (mean \pm SD)

Female: male ratio

Disease duration (months) (mean $\pm \mathrm{SD})$

Disease activity

SLEDAI (mean \pm SD)

Renal SLEDAI (mean \pm SD)

Serum NGAL $(\mathrm{pg} / \mathrm{ml})$ (Median (IQR)

Urine NGAL $(\mathrm{pg} / \mathrm{ml})$ Median $(\mathrm{IQR})^{\star \star}$

$\begin{array}{lr}\text { Urine NGAL pg/mg_creatinine Median (IQR) } & 14100(11250-19650) \\ & 14743.7(10487.7-21051.8)\end{array}$

$\begin{array}{ccc}10.6 \pm 3.2 & 10.9 \pm 2.5 & 10.7 \pm 1.9 \\ 8.4 \pm 3.1 & 9.4 \pm 2.3 & 8.9 \pm 2.4 \\ 39: 15 & 41: 13 & 11: 3 \\ 23.7 \pm 32.4 & 16.6 \pm 23.1 & 14.5 \pm 19.2\end{array}$

$11: 3$

$20.9 \pm 6.6$

$2.1 \pm 1.0$

$12.5 \pm 2.7$

18245 (16137.5-21062.5)

$41875.0(36243.75-46575)$

$33288.65(24610.7-44243.9)$
$7.1 \pm 2.0$

18375 (14375.0-26142.5)

18475 (15287.5-22500)

$14541.2(11939.3-22043.08$
$11.0 \pm 2.0$

$18: 8$

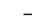

(16328.5-31143.8) 